\title{
On the Mathematical Modeling of Memristors
}

\author{
A. G. Radwan ${ }^{1,2}$, M. Affan Zidan ${ }^{1}$ and K. N. Salama ${ }^{1}$ \\ ${ }^{1}$ Electrical Engineering Program \\ King Abdullah University of Science and Technology (KAUST) \\ Thuwal, Kingdom of Saudi Arabia 23955-6900 \\ ${ }^{2}$ Department of Engineering Mathematics \\ Faculty of Engineering, Cairo University, Egypt \\ Email: \{ahmed.radwan, mohammed.zidan, khaled.salama\}@kaust.edu.sa
}

\begin{abstract}
Since the fourth fundamental element (Memristor) became a reality by HP labs, and due to its huge potential, its mathematical models became a necessity. In this paper, we provide a simple mathematical model of Memristors characterized by linear dopant drift for sinusoidal input voltage, showing a high matching with the nonlinear SPICE simulations. The frequency response of the Memristor's resistance and its bounding conditions are derived. The fundamentals of the pinched $i-v$ hysteresis, such as the critical resistances, the hysteresis power and the maximum operating current, are derived for the first time.
\end{abstract}

\section{INTRODUCTION}

The Memristor $(M)$ is believed to be the fourth fundamental two terminals passive element, beside the Resistor $(R)$, the Capacitor $(C)$ and the Inductor $(L)$. The existence of such element was postulated by Leon Chua in the seventies [1]. Recently a practical implementation of the Memristor using partially doped $T_{i} O_{2}$ was presented by Hewlett-Packard [2] as shown in Fig. 1, where $w \in(0, D)$. When the applied potential is removed the dopant boundary will remain at its location which will be the initial value for any later movements.

According to [2] the memristive property naturally appears in nanoscale devices, so the understanding of Memristance will improve the studying and modeling of nanodevices characteristic, which includes the current-voltage hysteresis behavior observed in many nanodevices. The invention of the Memristor was the key for postulating new elements by Chua such as the Memcapacitors and the Meminductors [3].

The Memristor found application in memory [4], biology [5], and spintronic [6]. Recently the Memristor was modeled using a linearized model of the pinched $i-v$ hysteresis as in [7] or qualitatively as in [8], [9]. However these efforts failed to capture many of its characteristics. A Memristors model for DC, square and triangular signals is presented in [10]. This model is used in the analysis of Memristor-based Wien-family oscillators [11].

The instantaneous resistance $R$ of the Memristor is given by [2],

$$
R=x \cdot R_{o n}+(1-x) R_{o f f}
$$

where $x=w / D, R_{o n}$ is the resistance of the completely doped Memristor, and $R_{o f f}$ is for the undoped Memristor. The speed dopant movement is defined as,

$$
\frac{d x}{d t}=k \cdot i(t) \cdot f(x)
$$
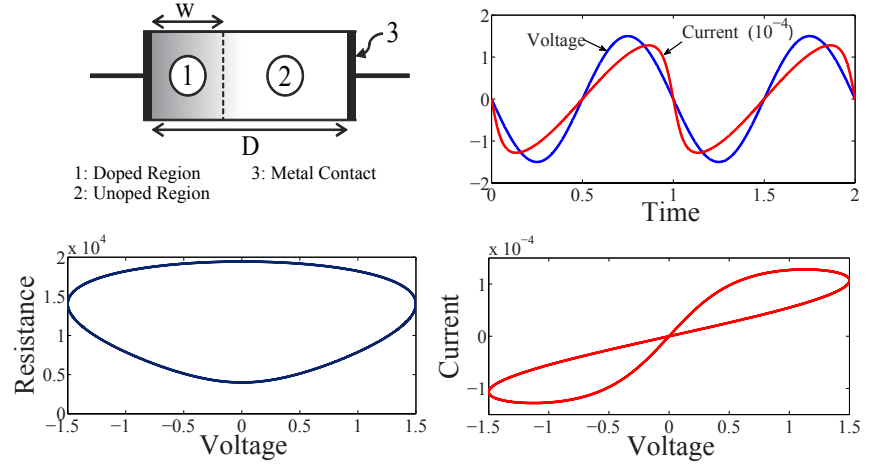

Fig. 1. The abstract structure of the HP's Memristor [2], current versus time, and current and resistance versus input voltage are plotted for sinusoidal wave input voltage of $1 \mathrm{~Hz}$ frequency and $-1.5 \mathrm{~V}$ peak voltage. Memristor's Parameters: $R_{o f f}=38 k \Omega$, Ron $=100 \Omega, R i=4 k \Omega$ and $p=10$.

where $f(x)=1-(2 x-1)^{2 p}$ is the window function for nonlinear dopant drift given in [12], and $k=\mu_{v} \cdot R_{o n} / D^{2}$. Fig. 1 shows some basic relationships between the current, voltage, and resistance for the HP's Memristor.

In this paper the frequency response of the Memristor's resistance is studied in the case of linear dopant drift for sinusoidal waveform for the first time. A condition on the ratio of $\left(v_{o} / f\right)$ to maintain unsaturated resistance is introduced. A simple implicit equation for the pinched $i-v$ hysteresis is derived, showing its general fundamentals such as critical resistances, maximum current magnitude and location, and the power under and inside this hysteresis. All mathematical equations are compared with the nonlinear Memristor's SPICE model given in [12], showing perfect matching.

\section{The Frequency Response of the Memristor's RESISTANCE}

The instantaneous resistance of the Memristor subjected to a sinusoidal input can be derived from equations (3) and (2), for linear dopant $f(x)$, and can be simplified as,

$$
R^{2}=R_{i}^{2}-\frac{2 V_{o} k R_{d}}{\pi f} \sin ^{2}(\pi f t), R \in\left(R_{o n}, R_{o f f}\right)
$$

where $k=\mu_{v} \cdot R_{o n} / D^{2}, \mu_{v}$ is dopant drift mobility, $f$ is the frequency of the input sinusoidal waveform, $R_{i}$ is the initial Memristor resistance at $t=0$, and $R_{d}=R_{o f f}-R_{o n}$ is 


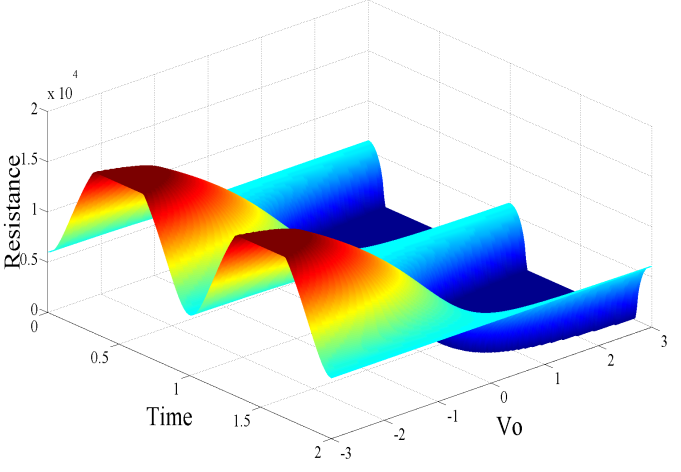

Fig. 2. Memristor's resistance versus time $-V_{o}$ plane for $1 \mathrm{~Hz}$ sinusoidal waveform with $f=1 \mathrm{~Hz}, R_{o f f}=16 k \Omega, R_{o} n=100 \Omega$, and $R i=6 k \Omega$.

the difference between the boundary resistances. However, the instantaneous resistance must be bounded by $R_{o} n$ and $R_{o} f f$.

The range of $R$ depends on the sign of $V_{o}$. Hence $R \in$ $\left[R_{i}, R_{o f f}\right)$ in case of $V_{o}<0$ or $\left(R_{o n}, R_{i}\right]$ for $V_{o}>0$. For any sinusoidal signal starting with a zero voltage, the Memristor's resistance reaches its maximum or minimum value at $t=$ $(2 n+1) T / 2 ; n=0,1,2, \cdots$, such that,

$$
R_{M}^{2}=R_{i}^{2}-\frac{2 V_{o} k R_{d}}{\pi f}, R_{M} \in\left(R_{o n}, R_{o f f}\right)
$$

The magnitude of $R_{M}$, whether maximum or minimum, depends on the sign of $V_{o}$. However, at $t=n T, n=0,1,2, \cdots$, the resistance returns to its initial value, $R=R_{i}$. Fig. 2 shows the 3D surfaces of the Memristor's resistance versus $t$ - $V_{o}$ plane.

The resistance has the shape of $\sin ^{2}(\cdot)$. However for high amplitude voltage there is clipping effect due to the bounding $R_{o f f}$ and $R_{o n}$. As $\left|V_{o}\right|$ increases the effect of the boundary resistances $R_{\text {off }}, R_{\text {on }}$ appears as clipping effect.

Fig. 3 shows the Memristor's resistance versus $v(t)$ - $f$ plane. Generally, the resistance clipping to $R_{o f f}$ exists at lower frequency (from the upper saturation plane). However, as frequency increases, the clipping interval decreases in time until it vanishes at a certain frequency. Any cross-section at fixed high frequency will show an ellipse shape, and the small

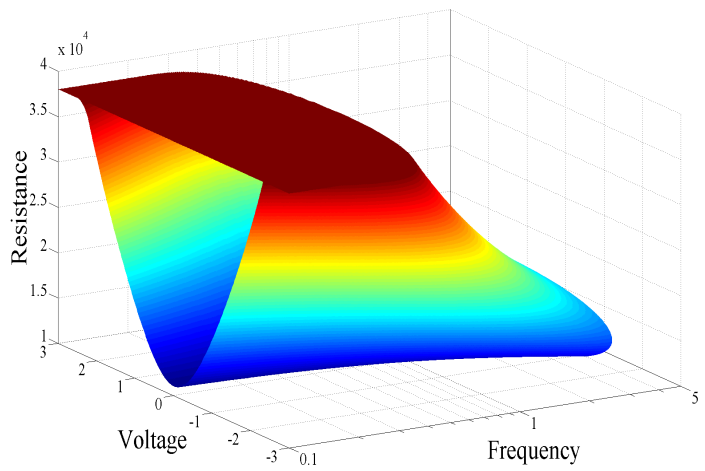

Fig. 3. Memristor's resistance versus a sinusoidal input and frequency for a peak input voltage of $3 \mathrm{~V}, R_{o f f}=38 k \Omega, R_{o} n=100 \Omega$, and $R i=11 k \Omega$.

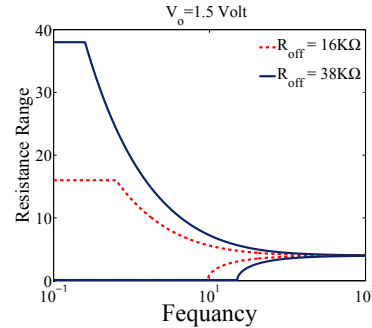

(a)

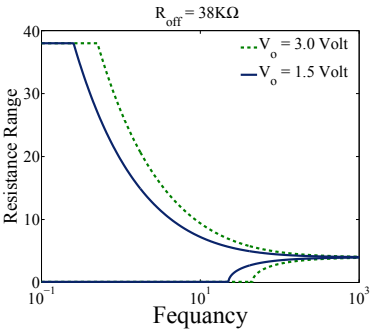

(b)
Fig. 4. Range of Memristor's resistance versus frequency, for sinusoidal wave input voltage for different (a) $R_{o f f}$, and (b) peak voltages $V_{o}$. Memristor's Parameters: $R_{o n}=100 \Omega, R_{i}=4 k \Omega$.

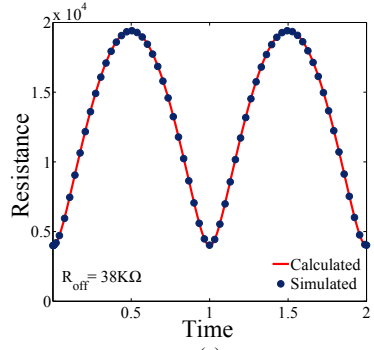

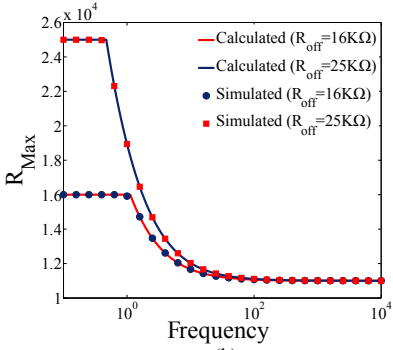

(b)
Fig. 5. Numerical and SPICE simulations in (a) time domain, with Memristor's Parameters: $R_{o n}=100 \Omega$, and $R_{i}=4 k \Omega$ (b) frequency domain, with Memristor's Parameters: $R_{o n}=100 \Omega, R_{i}=11 k \Omega$ and $p=10$.

axis of the ellipse area decreases and rotates as frequency increase.

Fig.4 shows the range of Memristor's resistance for both $\pm V_{o}$ for two different cases. Fig. 4(a) studies the effect of $R_{\text {off }}$ on the operating range of resistance. As $R_{\text {off }}$ decreases the Memristor's resistance saturates at $R_{o f f}$ for wider range of frequencies but it saturates at $R_{o n}$ for less range of frequencies. However, for fixed $R_{o} f f$ and two different values of $V_{o}$ as shown in Fig. 4(b), as $V_{o}$ increases both ranges of frequencies increase. Fig. 5(a) shows the the resistance given by equation (3) compared to the nonlinear Memristor's SPICE model given in [12].

Fig. 5(b) shows the maximum resistance of the transient SPICE simulation results for different frequencies chosen as 6 points/decade, while the solid lines are the calculated maximum frequencies according to equation (4). These simulations are repeated for two different cases showing identical matching with the SPICE model. Both the frequency and the voltage amplitude affect the resistance range and the rotation of the pinched $i-v$ hysteresis. The boundaries for non-saturating resistance are given by,

$$
\begin{aligned}
\left(V_{o} / f\right)_{o f f} & =\frac{\pi}{2 k R_{d}}\left(R_{i}^{2}-R_{o f f}^{2}\right)<0 \\
\left(V_{o} / f\right)_{o n} & =\frac{\pi}{2 k R_{d}}\left(R_{i}^{2}-R_{o n}^{2}\right)>0
\end{aligned}
$$

The difference between these boundaries, which is independent on $R_{i}$, is given by,

$$
\left|V_{o} / f\right|_{s a t}=\frac{\pi D^{2}}{2 \mu_{v}}\left(1+R_{o f f} / R_{o n}\right)
$$




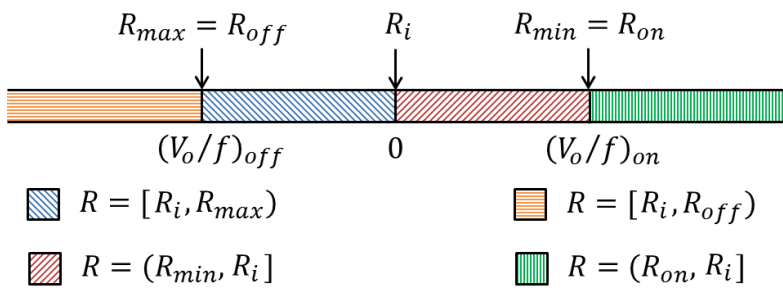

Fig. 6. Intervals of the Memristor's resistance versus $\left(V_{o} / f\right)$.

which is independent of $R_{i}$. This value is considered as the minimax ratio $\left|V_{o} / f\right|$ required for the Memristor's resistance to reach saturation, either $R_{o f f}$ or $R_{o n}$, when $R_{i}$ equals to $R_{o n}$ or $R_{\text {off }}$ respectively as shown in Fig. 6.

According to equation (4), the range of the Memristor's resistance depends on the value of $R_{M}$ which is inversely proportional to the frequency $(f)$. Thus the resistance range decreases with the increase of the frequency. Consequently the Memristor's current range decreases leading to a shrinkage of the pinched hysteresis. This will be further elaborated in the next section.

\section{Qualitative StUdy OF THE $i-v$ Hysteresis}

The 3D pinched $i-v$ hysteresis as a function of frequency is shown in Fig. 7. As the frequency increases, with same amplitude voltage, the pinched hysteresis shrinks and rotates. This figure describes the rotation only in very narrow band of frequencies from $0.5 \mathrm{~Hz}$ up to $2 \mathrm{~Hz}$. The dotted points in Fig. 8(a) shows the SPICE model pinched $i-v$ hysteresis for $V_{o}=-1.5$ and $f=1 \mathrm{~Hz}$. However the solid line represents the numerical simulation using the mathematical model at the same input data, showing the exact matching with the nonlinear SPICE model. In this section some of the main characteristics of the pinched $i$ - $v$ hysteresis such as maximum current, angle of rotation with frequency, and power inside the hysteresis are derived.

\section{A. Implicit Resistance Equation:}

The implicit equations which relate $R_{M}$ as function of $v$ is,

$$
R_{ \pm}=\sqrt{R_{i}^{2}-\frac{V_{o} k R_{d}}{\pi f}\left(1 \pm \sqrt{1-\left(v / V_{o}\right)^{2}}\right)}
$$

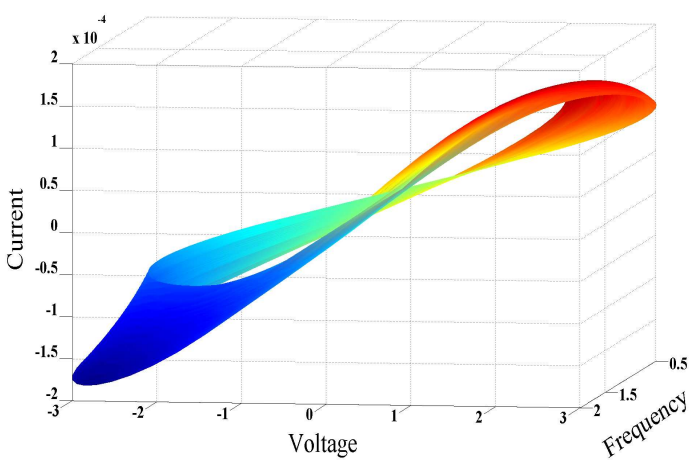

Fig. 7. Memristor's 3D $i$ - $v$ hysteresis for a sinusoidal input voltage.

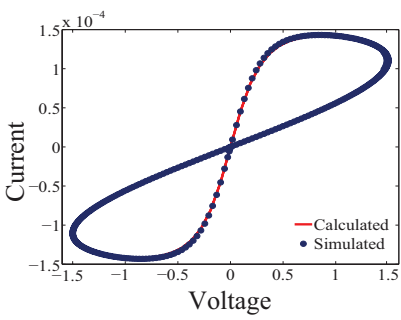

(a)

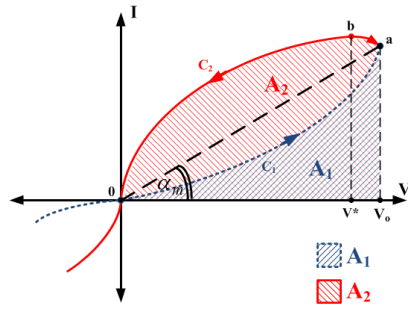

(b)
Fig. 8. (a) $i-v$ hysteresis at frequency of $1 \mathrm{~Hz}$ and peak voltage of -1.5 Volt using $R_{o f f}=16 k \Omega, R_{o n}=100 \Omega, R_{i}=4 k \Omega$ and $p=10$. (b) General schematic of the Memristor's pinched $i-v$ hysteresis.

where the positive sign is applied when the voltage increases and the negative when decreases. Thus the rate of change of $v$ must be known (ie memorize the previous value). Consequently, the implicit equations for the $i-v$ hysteresis is given by $i_{1,2}=v / R_{ \pm}$, which matches the SPICE simulations as shown in Fig. 8(a).

\section{B. Location of the peak current:}

The peak current, occurring at the point $b$ on the pinched $i-v$ hysteresis, is given by $i^{*}=v / R^{*}$, where $v^{*}$ and $R^{*}$ are the voltage and resistance at point $b$ as shown in Fig. 8(b). Let,

$$
y=\sqrt{1-\left(v / V_{o}\right)^{2}}, \alpha_{1}=R_{i n}^{2}-\frac{V_{o} k R_{d}}{\pi f}, \alpha_{1}=\frac{V_{o} k R_{d}}{\pi f}
$$

The critical point at which the maximum current occurs can be obtained by evaluating, $\partial i / \partial v=(\partial i / \partial y)(\partial y / \partial v)=0$, which can be simplified into the following quadratic equation,

$$
\begin{gathered}
y^{2} \pm 2 \frac{\alpha_{1}}{\alpha_{2}} y+1=0 \\
y=1-\frac{\pi f R_{i} n\left(R_{i}-R_{M}\right)}{V_{o} k R_{d}}=\frac{\pi f\left(R_{i}-R_{M}\right)^{2}}{2 V_{o} k R_{d}} \\
v^{*}=V_{o} \sqrt{1-y^{2}}, R^{*}=\sqrt{R_{i n} R_{M}}
\end{gathered}
$$

Fig. 9 shows the peak current values $i^{*}$ and its location $v^{*}$ versus the frequency for unsaturated conditions. The minimum value of the frequency is calculated by the maximum absolute value of the equation (5a) and (5b). In this case to avoid Memristor's resistance saturation the frequency must be greater than $33.7784 \mathrm{~Hz}$, when $V_{o}=3 \mathrm{~V}, R_{\text {in }}=3 \mathrm{~K} \Omega, R_{\text {on }}=100 \Omega$, and $R_{\text {off }}=16 K \Omega$.

\section{Angle of the $i$ - $v$ hysteresis at peak voltage}

As shown in Fig. 8(b), the angle $\alpha_{m}$ indicates the rotation of the hysteresis curve and is given by,

$$
\alpha_{m}=\cot ^{-1}\left(R_{a}\right)=\cot ^{-1} \sqrt{R_{i n} R_{M}}
$$

where $R_{a}$ is the resistance at the peak voltage $v=V_{o}$ as,

$$
R_{a}=\sqrt{\left(R_{i}^{2}+R_{M}^{2}\right) / 2}
$$

Fig. 10 plots the Memristor's resistance versus frequency. This angle depends on the initial resistance $R_{i}$ and the ratio 


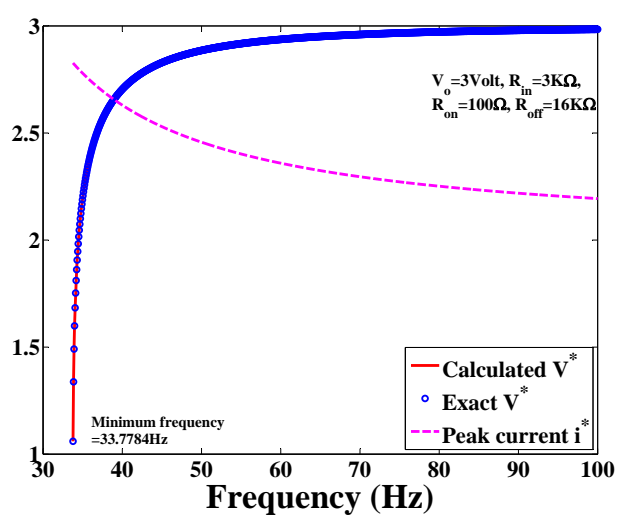

Fig. 9. Calculated peak current and its location $v^{*}$ and its exact value.

$V_{o} / f$ as discussed before. The maximum range of this angle is given by,

$$
\cot ^{-1}\left(R_{o f f}\right)<\alpha_{m}<\cot ^{-1}\left(R_{o n}\right)
$$

\section{$D$. Area enclosed by the $i-v$ hysteresis:}

The power under the curves $C_{1}$ and $C_{2}$, and the enclosed area inside the pinched hysteresis is a key characteristic of the Memristive device. The area inside one loop of the $i-v$ characteristic of the HP's Memristor is,

$$
A_{\text {Hysteresis }}=-\int_{C_{1}} i_{1} d-\int_{C_{2}} i_{2} d_{v}
$$

where the negative signs are due to the counterclockwise direction of the closed loop. The area under any curves between $v=v_{1}$ and $v_{2}$ can be calculated as,

$$
\begin{aligned}
A_{1} & =-\frac{2 \pi^{2} f^{2}}{k^{2} R_{d}^{2}} \int_{r_{1}=R_{i}}^{r_{2}=R_{a}}\left(R_{i}^{2}-\frac{V_{o} k R_{d}}{\pi f}-r^{2}\right) d r \\
& =\frac{2 \pi^{2} f^{2}}{3 k^{2} R_{d}^{2}}\left(R_{M}-R_{a}\right)\left(R_{a} R_{M}-R_{i}^{2}\right)
\end{aligned}
$$

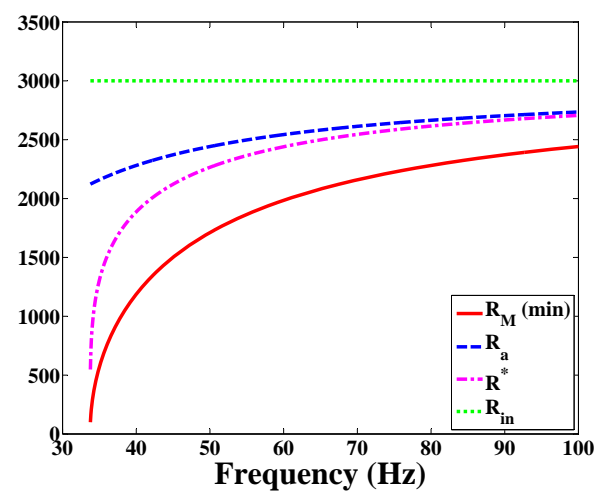

Fig. 10. The relationship between the initial resistance, resistance at peak voltage, resistance at peak current, and the minimum resistance versus frequency when $V_{o}=3 \mathrm{~V}, R_{i}=3 k \Omega, R_{o n}=100 \Omega$, and $R_{o f f}=16 k \Omega$.
In addition, $A_{2}$ can be given by,

$$
A_{2}=\frac{2 \pi^{2} f^{2}}{3 k^{2} R_{d}^{2}}\left(R_{a}-R_{i}\right)\left(R_{M}^{2}-R_{i} R_{a}\right)
$$

So, the hysteresis power (enclosed by the two curves) is,

$$
A_{\text {Hysteresis }}=A_{2}-A_{1}=\frac{\pi^{2} f^{2}}{3 k^{2} R_{d}^{2}}\left|R_{\text {initial }}-R_{M}\right|^{3}
$$

As the frequency increases by small amount, the difference between the $R_{i}$ and $R_{M}$ decreases, which compensates the $i$ - $v$ hysteresis area. This decreasing will be dominant, and this area at high frequency can be approximated as,

$$
A_{\text {Hysteresis }} \approx \frac{V_{o}^{3} k R_{d}}{3 \pi f R_{\text {initial }}^{3}}\left(1+\frac{V_{o} k R_{d}}{2 \pi f R_{\text {initial }}^{2}}\right)
$$

These expressions are valid if and only if $R \in\left(R_{o n}, R_{o f f}\right)$.

\section{CONCLUSION}

In this paper, we present a mathematical model for Memristors characterized by linear dopant drift under sinusoidal input voltage stimulation. The model describes the Memristor's resistance using its voltage and current without the need for the flux $(\phi)$ or charge equations $(Q)$. The frequency response of the Memristor's resistance and its bounding conditions are derived for the first time. A simple implicit equation for the pinched $i-v$ hysteresis is derived, describing its general characteristics such as critical resistances, maximum current magnitude and location, and the power inside this hysteresis. All the derived formulas are compared with the nonlinear Memristor's SPICE model showing perfect matching.

\section{REFERENCES}

[1] L. Chua, "Memristor-the missing circuit element," IEEE Transactions on Circuit Theory, vol. 18, no. 5, pp. 507-519, 1971.

[2] D. B. Strukov, G. S. Snider, and D. R. Stewart, "The missing memristor found," Nature, vol. 435, pp. 80-83, 2008.

[3] M. Di Ventra, Y. Pershin, and L. Chua, "Circuit elements with memory: Memristors, memcapacitors, and meminductors," Proceedings of the IEEE, vol. 97, no. 10, pp. 1717-1724, 2009.

[4] P. O. Vontobel, W. Robinett, P. J. Kuekes, D. R. Stewart, J. Straznicky, and R. S. Williams, "Writing to and reading from a nano-scale crossbar memory based on memristors," Nanotechnology, vol. 20, no. 42, p. 425204, 2009.

[5] Y. V. Pershin, S. La Fontaine, and M. Di Ventra, "Memristive model of amoeba learning," Phys. Rev. E, vol. 80, no. 2, p. 021926, 2009.

[6] Y. V. Pershin and M. Di Ventra, "Spin memristive systems: Spin memory effects in semiconductor spintronics," Phys. Rev. B, vol. 78, no. 11, p. 113309, 2008.

[7] D. Wang, Z. Hu, X. Yu, and J. Yu, "A PWL model of memristor and its application example," International Conference on Communications, Circuits and Systems, 2009. ICCCAS 2009, pp. 932-934, July 2009.

[8] F. Y. Wang, "Memristor for intrductory physics," Physics. class-ph, pp. $1-4,2008$.

[9] Y. N. Joglekar and S. J. Wolf, "The elusive memristor: properties of basic electrical circuits," European Journal of Physics, vol. 30, pp. 661-675, 2009.

[10] A. G. Radwan, M. A. Zidan, and K. N. Salama, "HP Memristor mathematical model for periodic signals and DC," 53rd IEEE International Midwest Symposium on Circuits and Systems (MWSCAS), pp. 861-864, Aug 2010

[11] A. Talukdar, A. G. Radwan, and K. N. Salama, "Time domain oscillating poles: Stability redefined in memristor based wien-oscillators," IEEE International Conference on Microelectronics (ICM), Dec 2010.

[12] Z. Biolek, D. Biolek, and V. Biolkova, "Spice model of memristor with nonlinear dopant drift," Radioengineering, vol. 18, no. 2, pp. 210-214, 2009. 